\title{
LA INCIDENCIA DE LA PARTICIPACIÓN COOPERATIVA EN ENTORNOS VIRTUALES
}

\section{EN EL RENDIMIENTO ACADÉMICO}

\section{THE IMPACT OF COOPERATIVE PARTICIPATION IN VIRTUAL ENVIRONMENTS}

\author{
IN ACADEMIC PERFORMANCE \\ Eneko Tejada Garitano; eneko.tejada@ehu.eus \\ Urtza Garay Ruiz; urtza.garay@ehu.eus \\ Ainara Romero Andoneg; ainara.romero@ehu.eus \\ Universidad del País Vasco
}

\section{RESUMEN}

El rendimiento de los equipos de trabajo depende de la calidad de las aportaciones que realicen. El objeto de esta investigación es conocer cómo incide la participación cooperativa en un entorno virtual en el rendimiento académico. Este estudio de carácter cuantitativo se llevó a cabo en la Escuela de Magisterio de Bilbao (UPV/EHU) sobre una muestra de 155 estudiantes del Grado en Educación Primaria. El alumnado conformó 25 equipos distribuidos según los roles de Belbin y trabajó de forma cooperativa y virtualmente por medio de wikis en el desarrollo de un proyecto educativo. De la investigación se extrae que los equipos y estudiantes que más participan en contexto virtual tienen mayor garantía de éxito académico.

PALABRAS CLAVE: participación virtual, aprendizaje colaborativo, roles de equipo, rendimiento académico.

\begin{abstract}
The performance of the students that constitute the work teams, depends on the roles that have been assigned to them and the contributions they make. The purpose of this research is to know how cooperative participation affects on a virtual environment in an academic performance. This quantitative study was carried out at the Teacher-training College of Bilbao (UPV / EHU) with a statistical sample of 155 students of Primary Education. The students formed 25 work teams distributed according to the roles of Belbin and worked cooperatively and virtually through wikis in order to develop an educational project. The research concludes that the teams and students who participate more in virtual context have further guarantee of academic success.
\end{abstract}

KEYWORDS: virtual participation, collaborative learning, team roles, academic performance. 


\section{INTRODUCCIÓN}

El trabajo cooperativo contribuye a que los estudiantes realicen una gestión más responsable y comprometida de su aprendizaje (Bellmunt, Torres, Bellmunt y Bellmunt, 2015), y a que generen nuevos conocimientos de mayor calidad (Johnson y Johnson, 2009; Tirado, Hernando, y Aguaded, 2016). Las investigaciones precedentes reflejan que esta forma de aprender en equipo mejora tanto el desempeño de tareas (Gavilán y Alario, 2012; Herman, 2013; Johnson, Johnson y Smith, 2014; Ning y Hornby, 2014), como el rendimiento académico (Prayekti, 2015; Vega, García y Vidal, 2013).

Las Wikis son un poderoso recurso de la web 2.0 que permiten trabajar en equipo (Biasutti y Heba, 2012; Cole, 2009). Fundamentalmente se caracterizan por contribuir a la participación activa de la construcción del conocimiento colectivo (Astall y Cowan, 2016; Villamor, 2007) y porque por medio de ellas los estudiantes crean redes de aprendizaje participativas y desarrollan habilidades de pensamiento crítico (Hazari, North y Moreland, 2009; Poyas, 2013; Su y Beaumont, 2010; Wake y Modla, 2012).

El éxito de esta herramienta reside en la calidad de las interacciones sociales que llevan a cabo sus participantes a la hora de trabajar virtualmente (Biasutti y El-Deghaidy, 2015). En ocasiones, el miedo a enjuiciar y reprobar el trabajo de los compañeros o viceversa provoca que la interacción colaborativa y las intervenciones sean de baja calidad y poco consistentes (Bennett, Bishop, Dalgarno, Waycott y Kennedy, 2012; Hadjerrouit, 2014; O’Bannon, Lubke, y Britt, 2013).

Para que el trabajo de los miembros de un equipo sea cooperativo, debe producirse una interdependencia positiva respecto a la tarea que realizan y a los incentivos que logran por medio de ella (Goikoetxea y Pascual, 2002; Slavin, 1983). Para ello, los equipos necesitan de un clima de confianza que les permita solventar los conflictos internos que provienen de su interacción (Ibarra, Rodríguez y Gómez, 2011; Johnson, Johnson y Hobulec, 1999). Y es que una mala configuración de conjunto puede llevar a malgastar la energía mental, física y emocional de forma innecesaria a sus integrantes (Rico, Alcover de la Hera y Tabernero, 2010).

El rendimiento de un equipo que trabaja cooperativamente, depende en buena medida de las funciones que llevan a cabo cada uno de sus miembros (Ramírez, Rodríguez y Blotto, 2016). Para que los resultados sean positivos, los conocimientos y habilidades de cada una de las unidades que lo componen deben converger (Mathieu, Maynard, Rapp y Gilson, 2008; Viles, Zárraga-Rodríguez y Jaca, 2013).

La teoría basada en los roles de equipo (Belbin, 2010), prevé la forma en que cada persona puede comportarse e interactuar dentro de un grupo de trabajo. Es decir, permite configurar equipos eficientes, sin que haya duplicidad de fortalezas y debilidades, y anticipar cuál puede ser su rendimiento (Belbin, 2010; Van de Water, Van de Water, y Bukman, 2007).

Los roles de equipo surgen de la combinación de los valores, actitudes y comportamientos de los individuos (Belbin, 2010) y se representan a través de tres grandes tipos de roles denominados mentales, sociales y de acción (Aritzeta, Swailes y Senior, 2007).

\begin{tabular}{|c|c|l|}
\hline Tipos de Rol & Roles específicos & \multicolumn{1}{c|}{ Características generales por las que se rigen } \\
\hline \multirow{2}{*}{ Mental } & Cerebro & Es creativo, imaginativo y poco dogmático \\
\cline { 2 - 3 } & Monitor-Evaluador & Perspicaz, serio y estratega. Percibe las diferentes \\
\hline
\end{tabular}




\begin{tabular}{|c|c|c|}
\hline & & opciones y situaciones. \\
\hline & Especialista & $\begin{array}{l}\text { Aporta conocimiento específico cuando trabaja en } \\
\text { equipo. }\end{array}$ \\
\hline \multirow{3}{*}{ Social } & Coordinador & $\begin{array}{l}\text { Suele mostrarse de forma madura y segura. A la hora } \\
\text { de realizar un trabajo colaborativo facilita la toma de } \\
\text { decisiones. }\end{array}$ \\
\hline & Cohesionador & $\begin{array}{l}\text { Funciona como elemento de ligazón dentro del } \\
\text { equipo. Aunque a veces infravalorado, es importante } \\
\text { porque es capaz de percibir las inquietudes de los } \\
\text { diferentes roles que forman el equipo y comportarse } \\
\text { de forma cooperadora. }\end{array}$ \\
\hline & $\begin{array}{l}\text { Investigador de } \\
\text { Recursos }\end{array}$ & $\begin{array}{l}\text { Destaca por su carácter comunicativo y extroversión. } \\
\text { En ocasiones es excesivamente optimista y su interés } \\
\text { depende de su entusiasmo. }\end{array}$ \\
\hline \multirow{3}{*}{ Acción } & Impulsor & $\begin{array}{l}\text { Es proactivo. Suele actuar de forma dinámica ante las } \\
\text { adversidades, sin embargo puede mostrarse agresivo } \\
\text { y generar conflictos dentro del quipo }\end{array}$ \\
\hline & Implementador & $\begin{array}{l}\text { Es disciplinado y valorados por su capacidad para } \\
\text { plasmar y concretar las ideas en acciones. }\end{array}$ \\
\hline & Finalizador & $\begin{array}{l}\text { Actúa de forma meticulosa y detallada en busca de } \\
\text { errores para corregir, lo que en ocasiones le lleva a } \\
\text { mostrarse de forma ansiosa. }\end{array}$ \\
\hline
\end{tabular}

Tabla 1: Roles.

Desde el punto de vista de los roles de Belbin los equipos que mejor rinden son los que se configuran de forma equilibrada, es decir, los que en el desarrollo de una tarea reúnen el máximo número de roles diferentes (Rico, Alcover de la Hera y Tabernero, 2010).

Para algunos autores el valor del equilibrio de un equipo respecto al resultado positivo que puede llegar a obtener no es del todo concluyente (Blenkinsop y Maddison, 2007; Jackson, 2002). Si bien algunas investigaciones constatan una relación entre el equilibrio de los equipos y su rendimiento (Aritzeta y Ayestaran, 2003; Senior, 1998), otras en cambio no (Chong, 2007; Van de Water, Ahaus y Rozier, 2008).

Teniendo en cuenta lo señalado, la intención de esta investigación es conocer cómo incide la participación cooperativa en un entorno virtual en el rendimiento académico.

\section{METODOLOGÍA}

Para la realización del estudio que se presenta a continuación se plantearon las siguientes preguntas de investigación:

1. ¿Qué tipo de roles de equipo son los que más participan?

2. ¿Los alumnos que más participan obtienen mejores resultados

académicos? 


\section{3. ¿Qué equipos de trabajo son los que mejor rendimiento obtienen?}

\section{Muestra}

El trabajo que se presenta a continuación se llevó a cabo en la Escuela de Magisterio de Bilbao (UPV/EHU) sobre una muestra de 155 alumnos y alumnas del Grado de Educación Primaria.

\section{Instrumentos}

En este estudio se ha aplicado una metodología cuantitativa, por lo que la recogida de datos se ha realizado de forma sistemática por medio de diferentes instrumentos.

El rendimiento académico se ha medido por medio de una prueba objetiva de conocimientos, y los resultados de los trabajos cooperativos llevados a cabo en las Wikis se han recogido por medio de una rúbrica de evaluación en la que se describen los niveles de calidad de las tareas realizadas.

Para obtener el rol con el que se identifica cada estudiante a la hora de trabajar de forma cooperativa se utilizó el cuestionario de roles de equipo de Belbin (Fraser y Neville, 1998). Los análisis estadísticos correspondientes a este estudio se han realizado con el programa SPSS (versión 22 para Windows), y para el estudio de los datos obtenidos se ha llevado a cabo un análisis de carácter descriptivo.

Las intervenciones que han realizado los estudiantes en contexto virtual en las wikis se han codificado teniendo en cuenta la media de intervenciones totales $(M=33,4)$.

\begin{tabular}{|c|c|c|c|c|}
\hline Muy baja & Baja & Media & Alta & Muy alta \\
\hline$<14$ & $\geq 14<28$ & $\geq 28<42$ & $\geq 42<56$ & $\geq 56$ \\
\hline
\end{tabular}

Tabla 2: Intervenciones.

\section{Procedimiento}

El conjunto de estudiantes desarrolló de forma cooperativa un proyecto para la asignatura de Didáctica General en el contexto virtual de las wikis. Los equipos de trabajo se diseñaron tomando como base los roles de Belbin. De esta manera, se tuvieron en cuenta las fortalezas y debilidades de cada uno de los nueve roles.

Los estudiantes fueron repartidos en 25 equipos de trabajo y cada grupo se diseñó atendiendo a la teoría de equilibrio de los roles de equipo, es decir, se configuró con el máximo de roles de equipo diferentes.

El proyecto realizado por el alumnado tuvo una estructura de recompensa grupal basada en el aprendizaje individual, por lo que la labor realizada por cada miembro del equipo repercutió en la valoración del trabajo grupal.

\section{RESULTADOS}

A continuación se presentan los resultados de la experiencia siguiendo el orden de las preguntas de investigación planteadas. En relación a la primera pregunta de investigación (1. ¿Qué tipo de 
alumnos son los que más participan?) el análisis de medias da como resultado que los roles de acción $(M=36,26)$ son los que más editan, seguido de los mentales $(M=32,67)$ y sociales $(M=29,33)$ respectivamente. La comparación de medias (ANOVA) de estos tres tipos de roles refleja que no hay diferencias significativas entre pertenecer a uno u otro $($ sig=0,08).

Tampoco se han encontrado diferencias significativas en la media de ediciones realizadas entre los equipos que tienen uno, dos o tres tipos de roles iguales $(\operatorname{sig}=0,775)$ o diferentes $(\operatorname{sig}=0,231)$ (Mental, Social, Acción).

Un estudio más detallado de los roles personales de Belbin muestra que los alumnos que se identifican con el rol de Finalizador son los que más han editado $(M=46,13)$ y por el contrario, los que menos son los que pertenecen al rol de Coordinador $(M=14,15)$. El análisis de las intervenciones de los nueves roles de Belbin da como resultado que hay diferencias significativas $(\operatorname{sig}=0,005)$ en la media de participación (muy baja, baja, media, alta y muy alta) entre estos dos roles.

\begin{tabular}{|c|c|c|c|}
\hline Tipo de rol & $\begin{array}{l}1 \text { Hombre } \\
2 \text { Mujer }\end{array}$ & $\begin{array}{l}1 \text { Aprobados } \\
2 \text { Suspendidos }\end{array}$ & Media \\
\hline \multirow{6}{*}{ Mental } & \multirow{3}{*}{1,0} & 1,0 & 16,00 \\
\hline & & 2,0 & 31,82 \\
\hline & & Total & 28,43 \\
\hline & \multirow{3}{*}{2,0} & 1,0 & 50,63 \\
\hline & & 2,0 & 15,80 \\
\hline & & Total & 37,23 \\
\hline \multirow{6}{*}{ Social } & \multirow{3}{*}{1,0} & 1,0 & 24,67 \\
\hline & & 2,0 & 18,89 \\
\hline & & Total & 20,28 \\
\hline & \multirow{3}{*}{2,0} & 1,0 & 34,78 \\
\hline & & 2,0 & 33,91 \\
\hline & & Total & 34,36 \\
\hline \multirow{6}{*}{ Acción } & \multirow{3}{*}{1,0} & 1,0 & 25,14 \\
\hline & & 2,0 & 18,00 \\
\hline & & Total & 21,57 \\
\hline & \multirow{3}{*}{2,0} & 1,0 & 43,48 \\
\hline & & 2,0 & 36,00 \\
\hline & & Total & 40,93 \\
\hline
\end{tabular}

Tabla 3. Media de la participación en el proyecto cooperativo virtual 
Desde el punto de vista del sexo las mujeres tienden a participar más que los hombres, ya que la media de edición de las mujeres $(M=37,56)$ supera a la de los hombres $(M=22,77)$ de forma significativa $(\operatorname{sig}=0,002)$.

\begin{tabular}{|c|c|c|c|c|c|l|l|l|c|}
\hline & Cer & Mon & Esp & Coord & In & Cohe & Imple & Impul & Fin \\
\hline $\begin{array}{c}\text { Cerebro } \\
\text { (Cer) }\end{array}$ & - & 1 & 1 &, 685 & - & 1 & 1 & 1 &, 968 \\
\hline Monitor evaluador (Mon) & 1 & - & 1 & 1,000 & - & 1 & 1 & 1 &, 982 \\
\hline Especialista (Esp) &, 685 & 1 & - &, 739 & - & 1 & 1 & 1 &, 875 \\
\hline Coordinador (Coord) & 1 & 1 &, 739 & - & - &, 315 &, 279 &, 452 &, 005 \\
\hline Investigador de recursos (In) & - & - & - & - & - & - & - & - & - \\
\hline Cohesionador (Cohe) & 1 & 1 & 1 &, 315 & - & - & 1 & 1 &, 406 \\
\hline Implementador (Imple) & 1 & 1 & 1 &, 279 & - & 1 & - & 1,000 &, 761 \\
\hline Impulsor (Impul) &, 968 & 1 & 1 &, 452 & - & 1 & 1 & - &, 993 \\
\hline Finalizador (Fin) & 1 &, 982 &, 875 &, 005 & - &, 406 &, 761 &, 993 & - \\
\hline
\end{tabular}

Tabla4. Comparación de medias de las intervenciones entre los roles de equipo.

Por otra parte, los estudiantes que han aprobado han editado una media de 37,34 veces y los que han suspendido 27,85 , y su diferencia es significativa (sig=0,038).

De esta forma, la media de intervenciones más alta la ha obtenido un perfil de alumnado de sexo mujer que ha aprobado y que se identifica con el rol de equipo mental $(M=50,63)$ (evaluador monitor).

En relación a la segunda pregunta de investigación (2. ¿Los alumnos que más participan obtienen mejores resultados académicos?) se observa que desde el punto de vista individual la calificación académica más alta (media $=6,08$ ) la ha obtenido el conjunto de estudiantes que ha realizado un número de participaciones dentro del rango alto $(\geq 42<56)$, seguido de los que han editado dentro del rango muy alto $(\geq 56)(M=5,4)$.

La comparación de medias del resultado académico individual del alumnado refleja que hay diferencias significativas $(s i g=0,039)$ entre los estudiantes que han editado muy poco $(<14)$ y bastante $(\geq 42<56)$. No obstante, como el número de ediciones correlaciona de forma leve con el resultado académico individual ( $R$ de Pearson= 0,171 ), no se puede afirmar que cuanto más editan los estudiantes mejor calificación académica personal obtienen.

Desde el punto de vista de los nueve roles de Belbin y el tipo de ediciones, los Implementadores (rol de acción) que han editado dentro del rango alto $(\geq 42<56)$ son los que han obtenido la media más alta $(M=9)$ de calificación académica individual. El rol mental de Especialista y el rol de acción Impulsor respectivamente, han obtenido las siguientes medias más altas de calificación $(M=7)$ y han editado también dentro del rango alto $(\geq 42<56)$. 
Desde el punto de vista del sexo los resultados académicos individuales más altos $(M=6,7)$ los han obtenido las mujeres que han editado dentro del rango alto $(\geq 42<56)$. Por su parte, los hombres que han obtenido la media de calificación más alta $(M=5,2)$, también han editado dentro del rango alto $(\geq 42<56)$.

Respecto a la tercera pregunta de investigación (3. ¿Qué equipos de trabajo son los que mejor rendimiento obtienen?) se observa que la nota media más alta $(M=7,35)$ la han obtenido los equipos de trabajo que tienen por lo menos cuatro roles diferentes y han realizado un número de intervenciones dentro del rango alto $(\geq 42<56$ ). No obstante, como el número de ediciones correlaciona de forma leve con el resultado del trabajo grupal ( $R$ de Pearson= 0,08 ), no hay relación directa entre el número de ediciones que ha realizado cada equipo y los resultados grupales que han obtenido.

\section{DISCUSIÓN Y CONCLUSIONES}

Una vez analizados los datos de la investigación se concluye que el número de ediciones que han realizado los estudiantes en el trabajo cooperativo virtual, no depende de que se identifiquen con un tipo de rol concreto, a pesar de que los estudiantes que se identifican con el rol de Finalizador (acción) editan significativamente más que los Coordinadores (social). Sin embargo, sí queda patente que los roles de acción son los que más han editado.

Desde la perspectiva de género, las mujeres y hombres que han participado con un número alto de ediciones han obtenido los resultados académicos individuales más positivos, siendo las mujeres las que significativamente mejor nota han obtenido. Esto de se debe a que la interacción social participativa resulta clave para lograr el éxito en el rendimiento académico de un contexto virtual como son las wikis (Biasutti y El-Deghaidy, 2015).

De este estudio también se desprende que los estudiantes que más editan en los trabajos virtuales cooperativos tienen mayor garantía de éxito individual. Por una parte se ha constatado que los roles de equipo que mejor calificación académica han obtenido, Implementador, Especialista e Impulsor respectivamente, han editado dentro del rango alto.

Por otra parte, queda de manifiesto la existencia de diferencias significativas en el rendimiento académico individual entre los estudiantes que han participado de forma intensiva y débil en cuanto al número de ediciones en la wiki. Y es que los estudiantes que más han editado, además de obtener un rendimiento académico superior respecto a los que han participado muy poco, también han aprobado significativamente más.

Si bien los resultados de la investigación ponen de manifiesto que los estudiantes que contribuyen al trabajo cooperativo mejoran su conocimiento (Pons, Prieto, Lomeli, Bermejo y Bulut, 2014; Herman, 2013; Johnson, Johnson y Smith, 2014; Ning y Hornby, 2014) y su rendimiento individual (Arceo y Rojas, 2010; Prayekti, 2015; Peklaj, 2003; Slavin, 1990; Vega, García y Vidal, 2013), el estudio no permite concluir que a más participación, más aprendizaje y mejores resultados académicos. Es decir, el número de participaciones no influye directamente en el aprovechamiento académico individual, ya que las veces que se ha editado y el resultado académico medio del alumnado correlacionan débilmente. Esto puede deberse a que la cantidad de aportaciones no se refleja la calidad de ellas. Desde el punto de vista de género las mujeres también tienden a participar más que los hombres de forma significativa. Esto se debe a que el tipo de demanda que requiere el trabajo realizado a través de la red en una Wiki, comunicación o 
actividad social, coincide con el tipo de actividad que principalmente realizan las universitarias cuando navegan por internet (Andreassen, Torsheim, Brunborg y Pallesen, 2012; Fernández-Villa, et., al, 2015).

Por último se concluye que a la hora de trabajar de forma cooperativa la diferencia de roles de equipo no repercute significativamente en el número de ediciones realizado y que los equipos que tienen por lo menos cuatro roles diferentes y han realizado un número de intervenciones dentro del rango alto son los que han obtenido la nota media grupal más alta. Sin embargo, esto no permite afirmar de forma categórica que los equipos que editan de forma intensiva obtienen mejores resultados académicos ya que las dos variables correlacionan de forma débil.

Desde la perspectiva de los roles de equipo, cuando los estudiantes participan cooperativamente en un entorno virtual de forma intensa, obtienen mejores resultados académicos y contribuyen a que sus equipos de trabajo rindan más.

Por último, se considera beneficioso profundizar en el tipo y calidad de aportaciones de los diferentes roles en contexto virtual, para fomentar el aprendizaje en equipos de trabajo en la universidad (Mathieu, Maynard, Rapp y Gilson, 2008).

\section{REFERENCIAS}

ANDREASSEN, C., TORSHEIM, T., BRUNBORG, G. y PALLESEN, S. (2012). Development of a Facebook addiction scale. Psychological Reports, 110, 1-17. Doi:10.2466/02.09.18. PR0.110.2.501-517

ARCEO, F. D. B. y ROJAS, G. H. (2010). Estrategias docentes para un aprendizaje significativo: una interpretación constructivista. McGraw Hill.

ARITZETA, S. A. y AYESTARÁN, A. A. (2003). Aplicabilidad de la teoría de los roles de equipo de Belbin: un estudio longitudinal comparativo con equipos de trabajo. Revista de psicología general y aplicada: Revista de la Federación Española de Asociaciones de Psicología, 56(1), 61-75.

ARITZETA, A., SWAILES, S. y SENIOR, B. (2007). Belbin's team role model: Development, validity and applications for team building. Journal of Management Studies, 44(1), 96-118. doi: 10.1111/j.1467-6486.2007.00666.x

ASTALL, C. y COWAN, J. (2016). Experiences of Using Wiki as a Participatory Learning Tool in Teacher Education. American Journal of Educational Research, 4(6) 459-471. doi: 10.12691/education-4-6-4.

BELBIN, M. (2010) Management teams: Why they succeed or fail (3rd ed.). London: Heinemann.

BELLMUNT, T. V., TORRES, P. R., BELLMUNT, I. V. y BELLMUNT, A. V. (2015). Aprendizaje cooperativo, aprendizaje percibido y rendimiento académico en la enseñanza del marketing. Educación XX1, 20(1), pp. 277-297. doi: 10.5944/educXX1.11408.

BENNETT, S., BISHOP, A., DALGARNO, B., WAYCOTT, J. y KENNEDY, G. (2012). Implementing Web 2.0 technologies in higher education: A collective case study. Computers \& Education, 59(2), 524-534. doi: http://dx.doi.org/10.1016/j.compedu.2011.12.022 
BIASUTTI, M. y EL-DEGHAIDY, H. (2015). Interdisciplinary project-based learning: an online wiki experience in teacher education. Technology, Pedagogy and Education, 24(3), 339-355. doi: http://dx.doi.org/10.1080/1475939X.2014.899510

BIASUTTI, M. y HEBA, E. (2012). Using Wiki in teacher education: Impact on knowledge management processes and student satisfaction. Computers \&y Education, 59(3), 861-872. doi: http://dx.doi.org/10.1016/j.compedu.2012.04.009

BLENKINSOP, N. y MADDISON, A. (2007). Team roles and team performance in defence acquisition. Journal of Management Development, 26(7), 667-682. doi: http://dx.doi.org/10.1108/02621710710761298

CHONG, E. (2007). Role balance and team development: A study of team role characteristics underlying high and low performing teams. Journal of Behavioral and Applied Management, 8(3), 202.

COLE, M. (2009). Using Wiki technology to support student engagement: Lessons from the trenches. Computers \& Education, 52, 141-146. doi: http://dx.doi.org/10.1016/j.compedu.2008.07.003

FERNÁNDEZ-VILLA, T., ALGUACIL OJEDA, J., ALMARAZ GÓMEZ, A., CANCELA CARRAL, J., DELGADORODRÍGUEZ, M., GARCÍA-MARTÍN, M., JIMÉNEZ-MEJÍAS, E., LLORCA, J., MOLINA, A., ORTíZ MONCADA, R., VALERO-JUAN, L., \& MARTíN, V. (2015). Uso problemático de internet en estudiantes universitarios: factores asociados y diferencias de género. Adicciones, 27(4), 265-275. doi:http://dx.doi.org/10.20882/adicciones.751

FRASER, A. y NEVILLE, S. (1998). Formación de equipos de trabajo: una guía práctica. México: Panorama.

GAVILÁN, P. y ALARIO, R. (2012). Efectos del aprendizaje cooperativo en el uso de estrategias de aprendizaje. Revista Iberoamericana de Educación, 60, 1-15.

GOIKOETXEA, E. y PASCUAL, G. (2002). Aprendizaje cooperativo: bases teóricas y hallazgos empíricos que explican su eficacia. Educación XXI, (5) 227-247. doi: http://dx.doi.org/10.5944/educxx1.5.1.392

HADJERROUIT, S. (2014). Wiki as a collaborative writing tool in teacher education: Evaluation and suggestions for effective use. Computers in Human Behavior, 32, 301-312. doi: http://dx.doi.org/10.1016/j.chb.2013.07.004

HAZARI, S., NORTH, A. y MORELAND, D. (2009). Investigating pedagogical value of wiki technology. Journal of Information Systems Education, 20(2), 187-198.

HERMAN, K. (2013). The Impact of Cooperative Learning on student engagement: Results from an Intervention. Active Learning Higher Education, 14(3), 175-185. doi: http://dx.doi.org/10.1177/1469787413498035

IBARRA, M.S., RODRÍGUEZ, G. y GÓMEZ, M. A. (2011). La evaluación entre iguales: beneficios y estrategias para su práctica en la universidad. Revista de Educación, 359, 206-231. doi: 10.4438/1988-592X-RE-2011-359-092

JACKSON, C. (2002). Predicting teamperformance froma learning processmodel. Journal of Managerial Psychology, 17(1), 6-13. doi: http://dx.doi.org/10.1108/02683940210415898 
JOHNSON, D. W., JOHNSON, R. T. y HOLUBEC, E. J. (1999). El aprendizaje cooperativo en el aula. Buenos Aires: Paidós.

JOHNSON, D. W., JOHNSON, R. T. y SMITH, K. A. (2014). Cooperative Learning: Improving University Instruction by Basing Practice on Validated Theory. Journal On Excellence In College Teaching, 25(3,4), 85-118.

JOHNSON, D.W. y JOHNSON, R.T. (2009). Joining together: group theory and group skills (10a Ed.). Boston: Allyn y Bacon.

MATHIEU, J., MAYNARD, M. T., RAPP, T. y GILSON, L. (2008). Team effectiveness 1997-2007: A review of recent advancements and a glimpse into the future. Journal of management, 34(3), 410-476.

NING, H. y HORNBY, G. (2014). The Impact of Cooperative Learning on tertiary EFL Learner's Motivation. Educational Review, 66(1), 108-124. doi: 10.1080/00131911.2013.853169

O'BANNON, B. W. LUBKE, J. K., y BRITT, V. G. (2013). You still need that face-to-face communication: Drawing implications from preservice teachers' perceptions of wikis as a collaborative tool. Technology, Pedagogy and Education, 22(2), 135-152. doi: http://dx.doi.org/10.1080/1475939x.2012.755470

PEKLAJ, C. (2003). Gender, Abilities, Cognitive Style and Student's achievement in Cooperative Learning. Psiholoskaobzorja/Horizons of Psychology, 12(4), 9-22.

PONS, R., PRIETO, M., LOMELI, C., BERMEJO, M. y BULUT, S. (2014). Cooperative Learning in Mathematics: a Study on the Effects of the parameter of equality on academic performance. Anales de Psicología, 30(3), 832-840. doi: http://dx.doi.org/10.6018/analesps.30.3.201231

POYAS, Y. (2013). Private path, public route: A multicultural group of teachers experiences Wikiassisted learning. Technology, Pedagogy and Education, 22(2), 153-172. doi: http://dx.doi.org/10.1080/1475939x.2013.778456

PRAYEKTI, M. (2015). The Influence Of Cooperative Learning Type STAD Vs Expository And Cognitive Style On Learning Of Comprehension Physics Con-cept In Among Students At Tenth Grade Senior High School In East Jakarta, Indonesia. Pinnacle Educational Research an Education, 3(3), 1-9.

RAMÍREZ, S. M. RODRÍGUEZ, J., y BLOTTO, B. (2016). El equipo de trabajo como estrategia de aprendizaje. InterCambios. Dilemas y transiciones de la Educación Superior, 3(1), 70-79.

RICO, R., ALCOVER DE LA HeRA, C. M. y TABERNERO, C. (2010). Efectividad de los equipos de trabajo: Una revisión de la última década de investigación (1999-2009). Revista de Psicología del Trabajo y de las Organizaciones, 26(1), 47-71.

SENIOR, B. (1998). An empirically-based assessment of Belbin's team roles. Human Resource Management Journal, 8 , 54-60. doi: http://dx.doi.org/10.1111/j.17488583.1998.tb00173.x

SLAVIN, R.E. (1983). When does cooperative learning increase student achievement? Psychological Bulletin, 94, 429-445. doi: http://dx.doi.org/10.1037/0033-2909.94.3.429 
SLAVIN, R.E. (1990). Cooperative learning. Theory, research and practice. New Jersey: Prentice Hall, Englewood Cliffs.

SU, F. y BEAUMONT, C. (2010). Evaluating the use of a wiki for collaborative learning. Innovations in Education and Teaching International, 47(4), 417-431. doi: http://dx.doi.org/10.1080/14703297.2010.518428

TIRADO, R., HERNANDO, Á. y AGUADED, J. I. (2016). Aprendizaje cooperativo on-line a través de foros en un contexto universitario: un análisis del discurso y de las redes. Estudios sobre Educación, 20, 49-71.

VAN DE WATER, H., AHAUS, K. y ROZIER, R. (2008). Team roles, team balance and performance. Journal of Management Development, 27(5), 499-512. doi: http://dx.doi.org/10.1108/02621710810871817

VAN DE WATER, T., VAN DE WATER, H. y BUKMAN, C. (2007). A balanced team generating model. European Journal of Operational Research, 180, 885-906. doi: http://dx.doi.org/10.1016/j.ejor.2006.04.017

VEGA, M., GARCÍA, M. y VIDAL, D. (2013). Avances acerca de los efectos del aprendizaje cooperativo sobre el logro académico y las habilidades sociales en relación con el estilo cognitivo. Revista Colombiana de Educación, 64, 155-174. doi: http://dx.doi.org/10.17227/01203916.64rce155.174

VILES, E., ZÁRRAGA-RODRÍGUEZ, M. y JACA, C. (2013). Tool to assess teamwork performance in higher education. Intangible Capital, 9(1), 281-304.

VILLAMOR, J. D. V. (2007). Usos didácticos del wiki en educación secundaria. Ikastorratza, eRevista de Didáctica, (1), 1-6.

WAKE, D. G. y MODLA, V. B. (2012). Using wikis with teacher candidates: Promoting collaborative practice and contextual analysis. Journal of Research on Technology in Education, 44(3), 243-265. doi: http://dx.doi.org/10.1080/15391523.2012.10782589

Para citar este artículo:

TEJADA, E.; GARAY, U. \& ROMERO, A. (2017). La incidencia de la participación cooperativa en entornos virtuales en el rendimiento académico. EDUTEC, Revista Electrónica de Tecnología Educativa, 60. Recuperado el dd/mm/aa de http://www.edutec.es/revista 